\title{
The Implication of Anthracosis in the Development of Pulmonary Adenocarcinoma
}

\author{
Mei Hou, Yukio Morishita, Tatsuo Iijima, Kentaro Mase, Yuichi Dai, Shigeki Sekine and \\ Masayuki Noguchi ${ }^{1}$ \\ Department of Pathology, Institute of Basic and Clinical Medical Sciences, University of Tsukuba, 1-1-1 \\ Tennodai, Tsukuba-shi, Ibaraki 305-8575
}

\begin{abstract}
The relationship between anthracosis, which is the deposition of black dust matter in the lung parenchyma, and the development of pulmonary adenocarcinoma has not been fully characterized. In order to clarify whether background black dust matter deposition could be implicated in the development of pulmonary adenocarcinoma, we measured the level of anthracosis at autopsy in 47 patients who had died of pulmonary adenocarcinoma. Both lungs of all 47 cadavers were examined. Twenty-micrometer sections were cut from formalin-fixed, paraffin-embedded blocks of the largest cut surface of each lung. Black dust matter was extracted from the sections and blotted onto a nitrocellulose membrane. The density of the blotted black dust matter was then analyzed using an imaging densitometer. There were no significant differences in the density of black dust matter deposition between lungs affected by pulmonary adenocarcinoma and control lungs. However, well differentiated adenocarcinomas tended to develop more frequently than poorly differentiated ones in lungs showing less deposition. We found a very strong correlation between the degree of black dust matter deposition and smoking history. Patients with severe anthracosis tended to have a poorer prognosis than those with mild anthracosis.
\end{abstract}

Key words: Black dust matter — Anthracosis — Lung adenocarcinoma — Differentiation — Smoking history

Although adenocarcinoma is the most common form of lung cancer in Japan, the U.S. and some other countries, and a gradual increase in its incidence has been recognized, ${ }^{1,2)}$ the mechanism by which it arises is not well understood. It has been said that the increase in lung adenocarcinoma since the 1950s correlates more with changes in smoking behavior and cigarette design than with diagnostic advances. ${ }^{3)}$ However, the association between adenocarcinoma and tobacco smoking remains unclear compared with that observed with squamous cell or small cell lung carcinomas. ${ }^{4-6)}$

Among the various DNA-damaging carcinogens, such as mycotoxins, plant alkaloids, nitrosamines, heterocyclic amines and polycyclic aromatic hydrocarbons (PAHs), PAHs are of particular interest as lung carcinogens because they are produced during the combustion of tobacco and are contained in diesel exhaust. ${ }^{7-9)}$ They are associated with many respirable environmental particulate pollutants. Insoluble respirable particles which produce black dust matter deposition in the lung are major components of tobacco smoke and diesel exhaust, and these are important sources of inhaled PAHs. ${ }^{10,11)}$ PAHs associated with insoluble respirable particles are retained in the lungs for long periods of time, ${ }^{12)}$ and compounds retained in the lungs can be metabolized there. Therefore, the grade of anthracosis (the amount of black dust matter

\footnotetext{
${ }^{1}$ To whom all correspondence should be addressed.
}

deposited in the lung) is a simple but good indicator for estimating exposure to insoluble carcinogens. However, anthracosis has usually been graded subjectively as mild, moderate, or severe during macroscopic examination of resected or autopsied lungs. Therefore, it is difficult to study the relationship between black dust matter deposition and lung cancer in detail.

With recent advances in molecular biology, the concept of multistep carcinogenesis, involving by an accumulation of multiple genetic alterations, has been proposed. ${ }^{13-15)}$ Many chemical carcinogens such as PAHs have been verifed to have mutagenic and carcinogenic properties and are thought to be associated with genetic alterations occurring early in the course of lung carcinogenesis. ${ }^{16,17)}$ However, there are very few reports examining the grade of exposure to chemical carcinogens and the histogenesis of human carcinomas. In this study, we measured the grade of background anthracosis in autopsied patients as an indicator of their exposure to chemical carcinogens, and examined the relationship between the grade of anthracosis and the histologic and clinicopathologic characteristics of their pulmonary adenocarcinomas.

\section{MATERIALS AND METHODS}

Patients Forty-seven patients who had died of pulmonary adenocarcinoma at the University Hospital of Tsukuba (Ibaraki) between 1982 and 1996 were examined. Their 

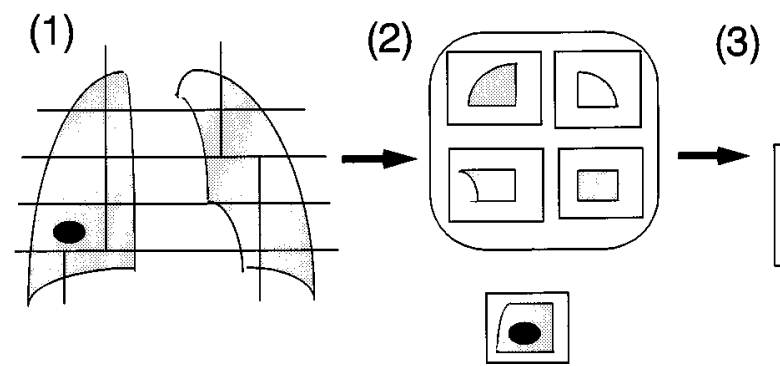

(3)

non-tumor tumor

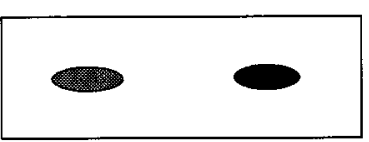

Fig. 1. Black dust matter and DNA were separately extracted from sections of background lung and tumorous lesions. (1) Both lungs were sliced along the largest cut surface and paraffin-embedded blocks were made from all the sliced tissues. All the blocks were cut into 20- $\mu$ m-thick sections. (2) All the sections were deparaffinized with xylene and dehydrated with ethanol. The dehydrated tissues were digested with proteinase $\mathrm{K}(100 \mu \mathrm{g} / \mathrm{ml})$ for 2 or 3 days at $48^{\circ} \mathrm{C}$. (3) After centrifugation and homogenization, the appropriate volume of the homogenized black dust matter (about $5 \mu \mathrm{g}$ DNA) was dot-blotted onto a nitrocellulose membrane and the density of the blotted carbon black was analyzed.

autopsied lungs were fixed with $10 \%$ formalin. For comparison, the autopsied lungs of 24 patients who had died of causes other than lung carcinoma were used after matching these patients with those who had died of pulmonary adenocarcinoma with respect to the ratio of males and females, and the average age. Both lungs were sliced along the largest cut surface and paraffin-embedded blocks were made from all the sliced tissues. These were cut into $20-\mu \mathrm{m}$-thick sections.

Extraction of black dust matter and DNA Black dust matter and DNA were separately extracted from sections of background lung and tumorous lesions, and the black dust matter was blotted onto a nitrocellulose membrane (Fig. 1). All the 20- $\mu$ m-thick sections were deparaffinized with xylene and dehydrated with ethanol. The dehydrated tissues were digested with proteinase $\mathrm{K}(100 \mu \mathrm{g} / \mathrm{ml})$ for 2 or 3 days at $48^{\circ} \mathrm{C}$. Black dust matter was separated by centrifugation for $40 \mathrm{~min}$ at $5000 \mathrm{rpm}$. DNA was extracted with phenol and chloroform, as described previously. ${ }^{18)}$ The centrifuged black dust matter was washed with phosphate buffer and homogenized using an UltraTurrax homogenizer (IKA, Stanfer, Germany). The black dust matter was suspended in a buffer (1× Tris-EDTA) and the appropriate volume of the suspension was dotblotted onto a nitrocellulose membrane using "Hybri-Slot" (Gibco BRL, Gaithersburg, MD). The amount of sample loaded on each lane corresponded to tissue from which 5 $\mu \mathrm{g}$ of DNA had been extracted. The density of the blotted black dust matter was analyzed using a GS-700 imaging densitometer (Bio Rad, Hercules, CA).

Statistical analysis All statistical analyses were performed using Fisher's exact probability test.

\section{RESULTS}

Light-microscopic examination revealed black dust matter deposits mainly in the subpleural, peribronchial, perivascular and fibrotic regions of the lung (Fig. 2). We found a good correlation between the macroscopic grading of anthracosis and the absolute absorbance $(A)$ value detected by densitometry (Fig. 3).

First, we compared the mean $A$ value for the lungs of the patients with pulmonary adenocarcinoma with that of the control patients with non-cancerous diseases. The mean $A$ values of the pulmonary adenocarcinoma group (47 patients) and the control group (24 patients) were $0.45 \pm 0.057$ and $0.43 \pm 0.071$, respectively. The difference was not significant. Next, we subdivided the patients according to tumor differentiation into groups with well, moderately and poorly differentiated adenocarcinoma (Table I). The absolute $A$ values were also subdivided into three groups: (1) less than 0.3 , (2) 0.3 to 0.6 and (3) more than 0.6. There were significant differences in the deposition of black dust matter between the groups with differing degrees of differentiation. The mean $A$ value for black dust matter in patients with moderately differentiated adenocarcinoma was similar to that in control patients (Table I). However, the $A$ value in the patients with well differentiated adenocarcinoma was significantly lower than that in the control group. Conversely, the mean $A$ value for black dust matter deposition in the patients with poorly differentiated adenocarcinoma was higher than that in the control group.

The relationship between the degree of anthracosis and smoking history is shown in Table II. As expected, almost all heavy smokers (20 cigarettes/day or more) had severely anthracotic lungs. However, there were many patients with severely anthracotic lungs who were not heavy smokers (less than 20 cigarettes/day) and patients with moderately anthracotic lungs who were non-smokers.

The relationship between the degree of anthracosis and the time between tumor detection and death (survival time) was also examined. We divided the survival time into two groups: less than 6 months and 6 months or 

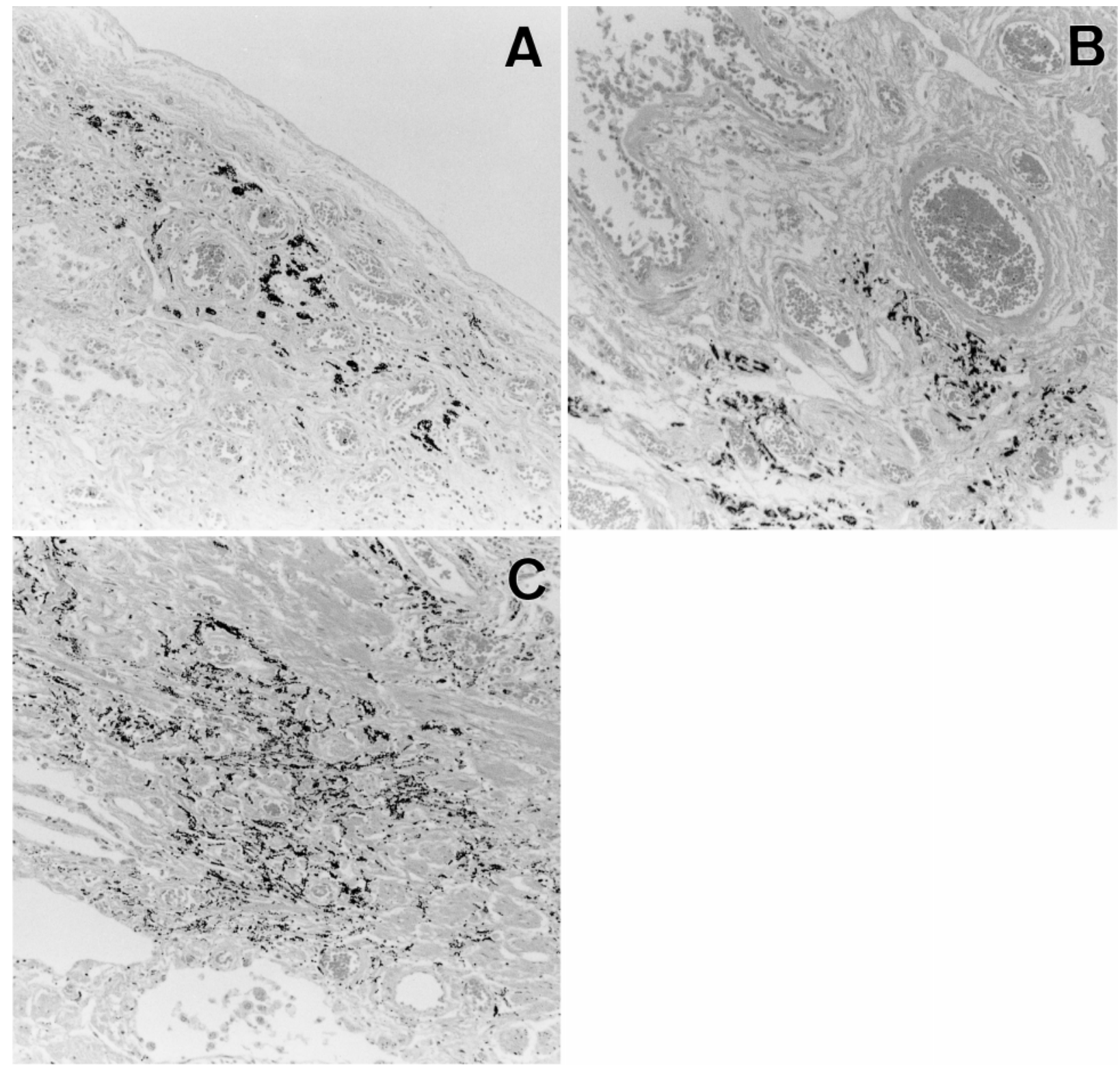

Fig. 2. Histology of black dust matter deposition in the lung. Black dust matter was mainly deposited in the (A) subpleural, (B) perivascular and peribronchial, and (C) fibrotic regions of the lung. (Hematoxylin and eosin, $\times 400)$

more. Patients with more anthracosis had a significantly shorter survival time than those without severe anthracosis $(P<0.05)$ (Table III).

\section{DISCUSSION}

Although anthracosis provides a simple measure of air pollution and cigarette smoking, ${ }^{12)}$ the objective estimation of the degree of black dust matter deposition has up to now been very difficult. Therefore, little work has been done on the relationship between the grade of anthracosis and the clinicopathological characteristics of pulmonary adenocarcinomas. For this study, we have developed an objective method for the quantitative evaluation of black dust matter deposition in autopsied lungs. Formalin-fixed, and paraffin-embedded sections were made from the largest cut surface of both lungs, then black dust matter was extracted from the blocks together with DNA and dotted onto a nitrocellulose membrane to allow measurement of the absorbance. Although this method is very simple, it is 


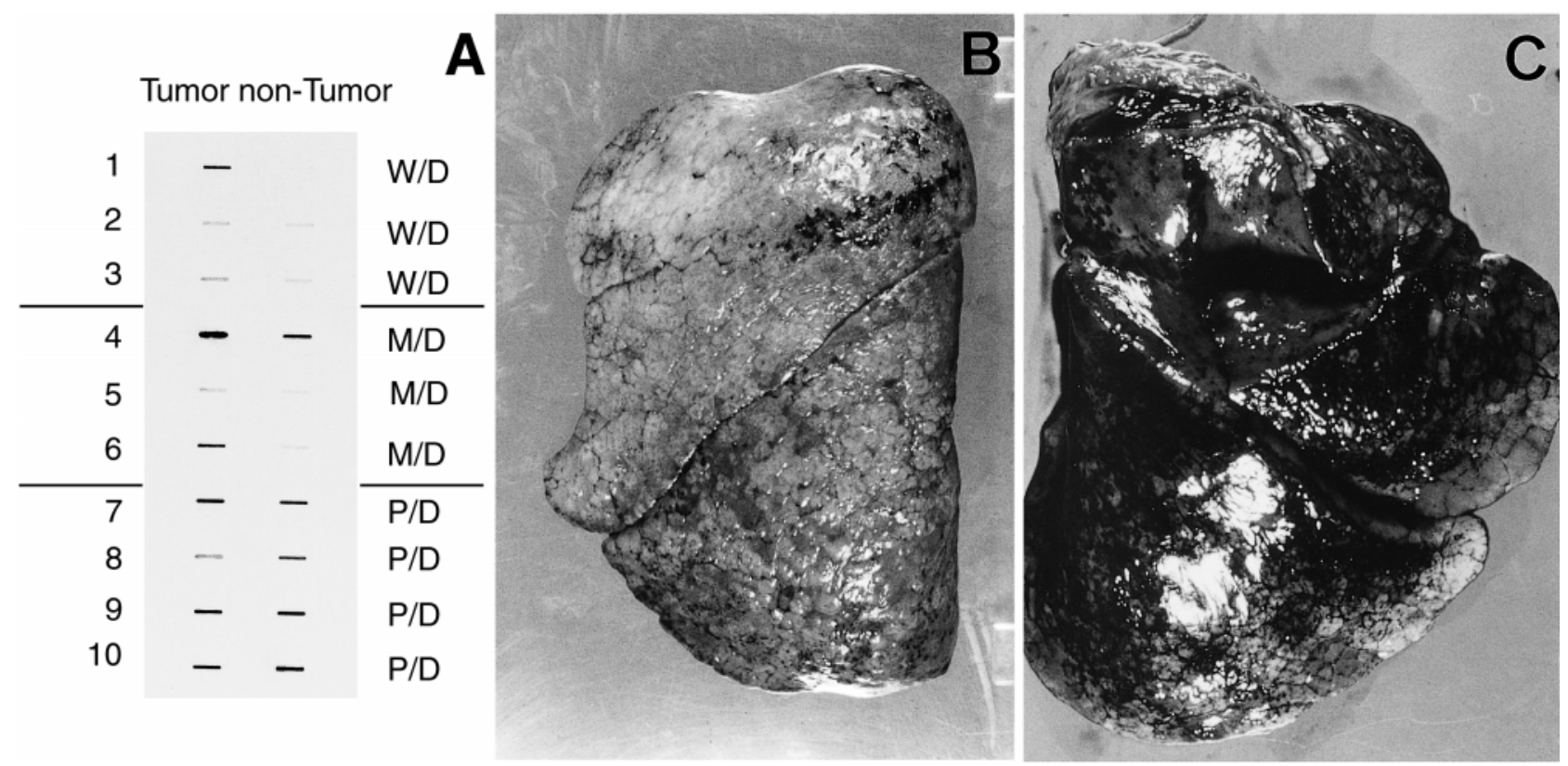

Fig. 3. (A) Representative results of the black dust matter dot-blotting. W/D, well differentiated; M/D, moderately differentiated; P/D, poorly differentiated; 1-10, patients' numbers. (B) Macroscopic appearance of lungs from patient $1(A: 0.000)$ and $(\mathrm{C})$ patient $10(A$ : 0.937).

Table I. Histological Differentiation, Black Dust Matter Deposition and Incidence of Smokers

\begin{tabular}{|c|c|c|c|c|c|}
\hline \multirow{2}{*}{ Differentiation } & \multicolumn{4}{|c|}{ Black dust matter deposition } & \multirow{2}{*}{$\begin{array}{l}\text { Incidence of } \\
\text { smokers }(\%)\end{array}$} \\
\hline & $<0.3^{\text {a) }}$ & $0.3-0.6$ & $>0.6$ & Mean $\mathrm{A} \pm \mathrm{SE}$ & \\
\hline Well diff. & $10^{\mathrm{b})}$ & 3 & 1 & $0.227 \pm 0.079^{c, \mathrm{~d})}$ & 57 \\
\hline Mod. diff. & 7 & 1 & 9 & $0.451 \pm 0.086$ & 71 \\
\hline Poorly diff. & 3 & 4 & 9 & $0.656 \pm 0.066$ & 94 \\
\hline
\end{tabular}

Mod., moderately; diff., differentiated.
a) Absorbance $(A)$.
b) Number of patients.
c) $P<0.05$
d) $P<0.01$

Table II. Smoking History and Black Dust Matter Deposition

\begin{tabular}{lcccc}
\hline \hline \multirow{2}{*}{ Smoking history } & \multicolumn{3}{c}{ Black dust matter deposition } & \multirow{2}{*}{ Total } \\
\cline { 2 - 4 } & $<0.3^{\text {a) }}$ & $0.3-0.6$ & $>0.6$ & \\
\hline $\begin{array}{l}\text { Non-smokers } \\
\text { Smokers }\end{array}$ & $10^{\text {c) }}$ & 2 & 0 & 12 \\
$\quad$ & & & & \\
$\quad<20^{\text {b) } / \text { day }}$ & 10 & 4 & 9 & 23 \\
$\quad 20 /$ day & 0 & 2 & 10 & 12 \\
\hline
\end{tabular}

a) Absorbance $(A)$.

b) 20 cigarettes/day.

c) Number of patients.
Table III. Survival Time and Black Dust Matter Deposition

\begin{tabular}{ccccc}
\hline \hline \multirow{2}{*}{ Survival time } & \multicolumn{3}{c}{ Black dust matter deposition } & \multirow{2}{*}{ Total } \\
\cline { 2 - 4 } & $<0.3^{\text {a) }}$ & $0.3-0.6$ & $>0.6$ & \\
\hline$<6$ months & $3^{\text {b) }}$ & 4 & $13^{\text {c) }}$ & 20 \\
$\geq 6$ months & 17 & 5 & 5 & 27 \\
\hline
\end{tabular}

Survival time: the time between tumor detection and death. a) Absorbance $(A)$.

b) Number of patients.

c) Significant difference between groups: $P<0.05$.

very useful for examining the relationship between the grade of black dust matter deposition and the morphologic and clinical characteristics of adenocarcinomas of the lung.

We found no significant differences in black dust matter deposition between patients with pulmonary adenocarcinoma and control patients, but black dust matter deposition in the lung parenchyma tended to be more severe in patients with poorly differentiated adenocarcinoma than in those with well differentiated adenocarcinoma. Furthermore, it is very interesting that the degree of anthracosis was milder in the lungs of patients with well differentiated adenocarcinoma, but was conversely more severe in the lungs of patients with poorly differentiated adenocarcinoma than in controls. 
In this study, a significant association between anthracosis and poorly differentiated adenocarcinoma was demonstrated. There seems to be a close relationship between severely anthracotic lungs, which are presumed to have been exposed to many chemical carcinogens, and the development of poorly differentiated pulmonary adenocarcinoma. These findings confirm the hospital-based casecontrol study by Suzuki et al. ${ }^{6}$ ) They analyzed the risk of adenocarcinoma of the lung associated with cigarette smoking according to grade of differentiation, and reported that the association between current smokers and poorly differentiated adenocarcinoma was significantly higher than the association with well differentiated adenocarcinoma. However, in our study, certain patients were observed who had smoked very little but had heavy black dust depositions in the lung (Table II). This can be explained by the involvement of other environmental factors, such as inhalation of diesel exhaust. Therefore, we consider that the effects of environmental factors on pulmonary adenocarcinogenesis can be estimated more accurately and more reliably by our system for monitoring black dust deposition than they can from smoking history.

In contrast, the data in Tables I, II and III indicate that well differentiated adenocarcinomas developed in mildly anthracotic lungs and that survival time in this case is longer than for less differentiated adenocarcinoma. These findings demonstrate that severely anthracotic lungs are

\section{REFERENCES}

1) Tsugane, S., Watanabe, S., Sugimura, H., Urano, Y. and Matsubara, S. Recent trends in different histological types of lung cancer in Tokyo based on pathological autopsy records. Jpn. J. Cancer Res., 78, 162-169 (1987).

2) Noguchi, M. and Shimosato, Y. The development and progression of adenocarcinoma of the lung. In "Lung Cancer V,” ed. H. H. Hansen, pp. 131-142 (1994). Kluwer Academic Publishers, Boston, MA.

3) Thun, A. J., Lally, C. A., Flannery, J. T., Calle, E. E., Flanders, W. D. and Heath, C. W., Jr. Cigarette smoking and changes in the histopathology of lung cancer. J. Natl. Cancer Inst., 89, 1580-1586 (1997).

4) Lubin, J. H. and Blot, W. J. Assessment of lung cancer risk factors by histologic category. J. Natl. Cancer Inst., 73, 383-389 (1984).

5) Wynder, E. L. and Kabat, G. C. The effect of low-yield cigarette smoking on lung cancer risk. Cancer, 62, 12231230 (1988).

6) Suzuki, T., Sobue, T., Fujimoto, I., Doi, O. and Tateishi, R. Association of adenocarcinoma of the lung with cigarette smoking by grade of differentiation and subtype. Cancer Res., 50, 444-447 (1990).

7) Phillips, D. H. Fifty years of benzo(a)pyrene. Nature, 303, 468-472 (1983). not closely associated with the development of well differentiated and biologically less malignant adenocarcinoma. Several reports have indicated that moderately and poorly differentiated adenocarcinomas appear to develop from very well differentiated papillary adenocarcinoma. ${ }^{19,20)}$ Therefore, we speculate that underlying anthracosis may accelerate the malignant transition of some well differentiated adenocarcinomas to less differentiated lesions.

Many genetic and epigenetic alterations which are associated with pulmonary carcinogenesis and its malignant progression have been reported. In order to clarify the relationship between pulmonary adenocarcinogenesis and the genetic and/or epigenetic alterations, it is necessary to consider the underlying abnormalities. The results of the present study strongly suggest that it is important to examine the alterations separately in tumors that develop in lungs that are less anthracotic and in those that develop in lungs that are more anthracotic.

\section{ACKNOWLEDGMENTS}

This work was supported in part by a Grant-in-Aid for Cancer Research from the Ministry of Health and Welfare.

(Received June 8, 1998/Revised August 26, 1998/2nd Revised September 21, 1998/Accepted September 24, 1998)

8) Fraga, C. G., Chigenaga, M. K., Park, J.-W., Degan, P. and Ames, B. N. Oxidative damage to DNA during aging: 8hydroxy-2'-deoxyguanosine in rat organ DNA and urine. Proc. Natl. Acad. Sci. USA, 87, 4533-4537 (1990).

9) Appel, B. R., Guirguis, G., Kim, I.-S., Garbin, O., Fracchia, M., Flessel, C. P., Kizer, K. W., Book, S. A. and Warriner, T. E. Benzene, benzo(a)pyrene, and lead in smoke from tobacco products other than cigarettes. Am. J. Public Health, 80, 560-564 (1990).

10) Sun, J. D., Wolff, R. K. and Kanaplly, G. M. Deposition, retention and biological fate of inhaled benzo(a)pyrene absorbed onto ultrafine particles and as a pure aerosol. Toxicol. Appl. Pharmacol., 65, 231-244 (1982).

11) Sun, J. D., Wolff, R. K., Kanaplly, G. M. and McClellan, R. O. Lung retention and metabolic fate of inhaled benzo(a)pyrene associated with diesel exhaust particles. Toxicol. Appl. Pharmacol., 73, 48-59 (1984).

12) Seto, H., Ohkubo, T., Watanabe, N., Ohnishi, K., Suzuki, S., Koike, M., Nakamura, K. and Kawahara, Y. Black dust matter in human lung and causes of the deposition. Ann. Rep. Tokyo Metrop. Res. Lab. P. H., 45, 139-148 (1994).

13) Vogelstein, B., Fearon, E. R., Hamilton, S. R., Kern, S. E., Preisinger, A. C., Leppert, M., Nakamura, Y., White, R., Smits, A. M. M. and Bos, J. L. Genetic alterations during 
colorectal-tumor development. N. Engl. J. Med., 319, 525532 (1988).

14) Tsuda, H., Hirohashi, S., Shimosato, Y., Terada, M. and Hasegawa, H. Clonal origin of atypical adenomatous hyperplasia of the liver and clonal identity with hepatocellular carcinoma. Gastroenterology, 95, 1664-1666 (1988).

15) Sugimura, T. Multistep carcinogenesis. A 1992 perspective. Science, 258, 603-606 (1992).

16) Noguchi, M., Hirohashi, S., Hara, F., Kojima, A., Shimosato, Y., Shinkai, T. and Tsuchiya, R. Heterogenous amplification of myc family oncogene in small cell lung carcinoma. Cancer, 66, 2053-2058 (1990).

17) Nesnow, S., Ross, J. A., Stoner, G. D. and Mass, M. J. Mechanistic linkage between DNA adducts, mutations in oncogenes and tumorigenesis of carcinogenic environmen- tal polycyclic aromatic hydrocarbons in strain $\mathrm{A} / \mathrm{J}$ mice. Toxicology, 105, 403-413 (1995).

18) Swafford, D. S., Nikula, K. J., Mitchell, C. E. and Belinsky, S. A. Low frequency of alterations in p53, $\mathrm{K}$-ras and $m d m 2$ in rat lung neoplasms induced by diesel exhaust or carbon black. Carcinogenesis, 16, 1215-1221 (1995).

19) Nomori, H., Hirohashi, S., Noguchi, M., Matsuno, Y. and Shimosato, Y. Tumor cell heterogeneity and subpopulations with metastatic ability in differentiated adenocarcinoma of the lung. Histologic and cytofluorometric DNA analyses. Chest, 99, 934-940 (1991).

20) Noguchi, M., Morikawa, A., Kawasaki, M., Matsuno, Y., Yamada, T., Hirohashi, S., Kondo, H. and Shimosato, Y. Small adenocarcinoma of the lung. Histologic characteristics and prognosis. Cancer, 75, 2844-2852 (1995). 